\title{
Synthesis, swelling behavior and surface microstructure of poly(sodium acrylate) gels crosslinked by aluminum ions
}

Tomofumi Harada, Yumiko Hirashima, Atsushi Suzuki*, Motoaki Goto ${ }^{1}$, Naohisa Kawamura ${ }^{1}$, Masayuki Tokita ${ }^{2}$

Department of Materials Science, Yokohama National University, Yokohama, 240-8501 Japan,

${ }^{1}$ Saitama Daiichi Pharmaceutical Co., Ltd., Kasukabe, Saitama, 344-0057 Japan,

${ }^{2}$ Department of Physics, Kyusyu University, Fukuoka, 810-8560 Japan.

\begin{abstract}
We report the synthesis and some unique properties of polymer gels cross-linked by metal ions. They are made of poly(sodium acrylate) and aluminum ions. Cylindrical and thin plate gels were synthesized in glass molds by mixing poly(sodium acrylate) with aluminum hydroxide of various concentrations. The swelling ratio of the gels was measured by changing the solvent $\mathrm{pH}$ and adding $\mathrm{NaCl}$ with different concentrations. Each gel was found to swell at the first stage and shrink thereafter by repeated solvent exchanges; the swelling ratio ultimately returned to the initial one just after gelation. These features could be explained using a qualitative model based on the ion exchange and the formation of hydrogen bonding. A characteristic surface microstructure was observed on the dehydrated gels using an atomic force microscope. The microstructures were analyzed in terms of the autocorrelation function and the root-mean-square roughness. The surface microstructure of this system was characterized by the microdomains, the shape of which was found to depend strongly on the amount of aluminum ions and the gel thickness at gelation. It could be controlled not only by the concentration of the cross-linker but also by the dehydration condition.
\end{abstract}

Keywords: Poly(sodium acrylate) gel; Chelation; Swelling ratio; Hydrogen bonding; Dehydrated gels; Surface structure; AFM; Microdomain

\footnotetext{
* Corresponding author. Tel. \& fax: +81-45-339-3846

E-mail:suzuki@post.me.ynu.ac.jp (A. Suzuki)
} 


\section{Introduction}

A hydrogel is a three-dimensional polymer network containing a large amount of water. In the last quarter century, many studies have been conducted on the swelling properties of various hydrogels, particularly neutral polymer gels, such as polyacrylamide or poly $(N$ isopropylacrylamide) and weakly charged gels by sodium acrylate or acrylic acid [1-10]. These hydrogels are usually prepared by a free-radical polymerization of monomers with a small amount of cross-linker, such as $N, N^{\prime}$-methylene-bis-acrylamide. The fundamental properties of these chemically cross-linked gels have been considerably elucidated. They are of crucial importance as functional materials for future applications of hydrogels. Moreover, hydrogels can be used to understand the mechanism of living systems that have advanced flexibility and higher functional performance. For example, it was recently reported [11] that the swelling of polyelectrolyte gels occurs in many physiological systems and plays a crucial role in physiological processes. The swelling behavior of fully neutralized sodium polyacrylate gels was investigated in aqueous salt solutions to understand this process [12].

As for practical applications, on the other hand, hydrogels have been used as retainers of water and solutes (water-absorbable solids) in many industries [13]. For example, disposable diapers and sanitary napkins require the use of gels as super water absorbents, and gels play a vital role in the fields of medicine, foods, chemistry, agriculture, and other industries. For these industries, it is also crucial to understand the physical and the chemical principles that govern the swelling degree and its speed under individual environmental conditions. Nevertheless, experimental investigations on the fundamental properties of strongly ionized polyelectrolyte gels, such as the swelling behavior, mechanical properties, and network structure, have not been extensively reported.

In this paper, we synthesized poly(sodium acrylate) gels physically cross-linked by aluminum ions, which have been widely used in the medical fields, for example, as anti-inflammatory analgesic cataplasms. To our knowledge, there is no report on the fundamental properties of this 
kind of polyelectrolyte hydrogel, which is physically cross-linked through a chelation mechanism by metal ions. In order to understand some properties of the gel, two different experiments were conducted. One experiment was on the swollen states: the swelling ratio was measured in detail whenever the solvent was repeatedly exchanged. The other was on the dehydrated states: the characteristic surface microstructure was observed with an atomic force microscope (AFM). New experimental results on the swelling behavior by repeated solvent exchanges and a microphase separation during dehydration are presented here. They were obtained by varying the cross-linking density and the dehydrating conditions.

\section{Experimental procedure}

\subsection{Gel synthesis}

Polyelectrolyte hydrogels cross-linked physically by metal ions were made of sodium acrylate and aluminum ions. The gels were synthesized in two shapes, a cylinder and a plate, by mixing poly(sodium acrylate) (for main constituent, Wako; $18.1 \mathrm{wt} \%$ aqueous solution, and the average degree of polymerization ranges from 2,700 to 7,500 ,) with aluminum hydroxide $\mathrm{Al}(\mathrm{OH})_{3}$ (for crosslinker, Kyowa Chemical Industry) of various concentrations (between 0.5 and $8 w t \%$ of poly(sodium acrylate) solution) in an acidic condition. The solution was mixed with a spatula, sealed by a Parafilm to prevent evaporation, and left in air at room temperature (around $25^{\circ} \mathrm{C}$ ) for one week. Thin cylindrical gels were prepared in glass microcapillaries with an inner diameter of $1.46 \mathrm{~mm}(50 \mu \mathrm{l})$, and thin plate gels were prepared between two glass plates (slides) with a thin spacer (thickness between $0.08 \mathrm{~mm}$ and a few millimeters).

In the gel synthesized with $1 \mathrm{wt} \%$ of $\mathrm{Al}(\mathrm{OH})_{3}$, the ratio of a sodium acrylate monomer to an Al ion is $15: 1$; therefore, the maximum amount of $\mathrm{Al}(\mathrm{OH})_{3}$ to contribute to the cross-linking is $5 \mathrm{wt} \%$ if the coordination number per aluminum ion is ideally 3. 


\subsection{Measurements}

The diameter of the cylindrical gel was measured using an optical microscope apparatus equipped with a calibrated measuring gauge with an accuracy of $1 \mu \mathrm{m}$. The prepared gel was immersed into two different solvents at room temperature: $\mathrm{pH}$-controlled water (between $\mathrm{pH}=4$ and 10) by adding an $\mathrm{HCl}$ solution (to decrease the $\mathrm{pH}$ ) or an $\mathrm{NaOH}$ solution (to increase the $\mathrm{pH}$ ) into distilled-deionized water (pure water) and an $\mathrm{NaCl}$ solution with different concentrations (between 0.01 and $1 \mathrm{~mol})$. The solvent was repeatedly exchanged, and the diameter of the gel was measured just before the solvent was exchanged.

The microscopic image of the NIPA gel surface in water was determined using the tapping mode AFM (Digital Instruments, NanoScope III) [14] with a sharpened silicon cantilever of $125 \mu \mathrm{m}$ length with spring constant of $53 \mathrm{Nm}^{-1}$. The lowest possible set-point voltage was utilized in order not to risk unnecessary damage to the gel. The amplitudes of the drive signal applied to the cantilever oscillation are used in the range between 0.5 and $2 \mathrm{~V}$ (typically $1 \mathrm{~V}$ ). The tapping frequencies ranged from 220 to $320 \mathrm{kHz}$, and the scan rates ranged from 0.5 to $2 \mathrm{~Hz}$ (typically $1 \mathrm{~Hz}$ ). All images (256 samples/line $\times 256$ lines) were processed by first order flattening in order to remove the background slope and the contrast and brightness were adjusted. [14-17].

\section{Results and discussion}

\subsection{Swelling properties}

Figure 1 shows the changes in the swelling ratio of as-prepared poly(sodium acrylate) gel synthesized with $4 \mathrm{wt} \% \mathrm{Al}(\mathrm{OH})_{3}$ at room temperature when the solvent $(100 \mathrm{ml}$ of $\mathrm{pH}$-controlled water or $\mathrm{NaCl}$ aqueous solution) was repeatedly exchanged every 24 hours. In this figure, the degree of swelling is represented by the swelling ratio, $d / d_{0}$, where $d$ is the diameter of the gel and $d_{0}$ is the original diameter (the inner diameter $1.46 \mathrm{~mm}$ of the capillary in which the gel was synthesized). As is shown in Fig. 1(a), the swelling behavior depended on the $\mathrm{pH}$ and on the 
number of water exchanges; in the case of $\mathrm{pH}=4$, the $d / d_{0}$ increased rapidly in one day, hereafter decreased to the original $d / d_{0}=1$ (just after the gelation) in three days. In the cases of pure water and $\mathrm{pH}=7$, the $d / d_{0}$ continuously increased and saturated to around $d / d_{0}=6$. In the case of $\mathrm{pH}=10$, the $d / d_{0}$ continued to increase rapidly after three days to more than 10 times the size of the original diameter. However, after the fourth water exchange, many cracks appeared in the surface, and the gel did not retain its shape. It deteriorated and finally disappeared due to the destruction of the cross-linking. On the other hand, for the repeated exchange of the $\mathrm{NaCl}$ aqueous solution, the qualitative changes of $d / d_{0}$ were similar in spite of different concentrations, $1,0.1$, and $0.01 \mathrm{M}$, which are shown in Fig. 1(b); the $d / d_{0}$ increased rapidly in one day, hereafter slightly decreased, and approached to the respective equilibrium value after three days.

In order to confirm the stability of the diameter saturation after several days, a long-term relaxation experiment was conducted for the gels synthesized with 2,4 and $8 \mathrm{wt} \% \mathrm{Al}(\mathrm{OH})_{3}$. The results are shown in Fig. 2. In this experiment, the solvent $(100 \mathrm{ml})$ was repeatedly exchanged, and the diameter of the gel was measured at the point when the gel achieved its equilibrium diameter after the solvent was exchanged, but not at a regular interval. The time in the horizontal axis is, therefore, not particularly important, which is just a measure of the relaxation time. As is shown in Fig. 2, the $d / d_{0}$ of the gels synthesized with $4 \mathrm{wt} \% \mathrm{Al}(\mathrm{OH})_{3}$ (same as Fig. 1) decreased to almost $d / d_{0}=1$, regardless of the $\mathrm{pH}$ of the solvent (except $\mathrm{pH}=10$ ), with increasing the number of water exchanges. On the other hand, the $d / d_{0}$ decreased and approached the respective equilibrium value for the gel with $2 \mathrm{wt} \% \mathrm{Al}(\mathrm{OH})_{3}$, and it approached $d / d_{0}=1$ more rapidly for the gel with $8 \mathrm{wt} \%$ $\mathrm{Al}(\mathrm{OH})_{3}$. These results indicate that in the short-term relaxation (several days in Fig. 1) the gel could not reach its equilibrium in the water-exchange process; however, in the long-term relaxation, the repeated water exchange would cause the gel to shrink as a result of attractive forces. Only the gel with $8 \mathrm{wt} \% \mathrm{Al}(\mathrm{OH})_{3}$ could keep the gel state in the $\mathrm{pH}=10$ base solution. This is because the cross-linking density of the gel increased at gelation with increasing the amount of $\mathrm{Al}(\mathrm{OH})_{3}[18]$. 
According to the swelling theory [19], the larger the cross-linking density, the larger the shrinking force, which is strong enough to maintain the network structure against the larger positive osmotic pressure in the $\mathrm{pH}=10$ base solution.

\subsection{Origin of the two-step relaxation in swelling gels}

From the results of the swelling behavior, two relaxation processes were obtained as a result of repeated water exchanges. One was short (a few days), and the other, long (a few weeks). Just after the gel was immersed into the $\mathrm{pH}$-controlled water, the sample gel started swelling and became constant after a few days, since the osmotic pressure created by the counter ions trapped within the Donnan potential (swelling force) was large. In this process, the original counter ions $\mathrm{Na}^{+}$could be replaced by $\mathrm{H}^{+}$. In a recent study [20], it was reported that the carboxyl groups ($\mathrm{COOH})$ in the gel are largely dissociated $-\mathrm{COO}^{-}$plus $\mathrm{H}^{+}$even when the $\mathrm{pH}$ of the water decreases to 5.6. In those steady states, the carboxyl groups $(-\mathrm{COOH})$ in the gel are largely dissociated $-\mathrm{COO}^{-}$ plus $\mathrm{H}^{+}$. Therefore, the reason that the final swelling ratio of the strongly ionized NIPA/SA gel would be as small as $d / d_{0}=1$ is unclear. It could be assumed that some attractive force between polymer chains is created as a result of the repeated water exchanges, which would compensate for the positive osmotic pressure due to the ionization [18]. Among the several intermolecular interactions, the most probable candidate for the new attractive force might be hydrogen bondings between the two non-ionized carboxyl groups $(-\mathrm{COOH})$, which is a similar phenomenon to that reported recently on chemical gels [21]. This long relaxation time does not result from the collective network diffusion, since the present gel with a diameter of a few millimeters would usually swell or collapse within a few hours at most [22]. The ionization of the individual carboxyl groups, the exchange of the counter ions $\mathrm{Na}^{+}$by $\mathrm{H}^{+}$, and the formation of hydrogen bonding should independently or collectively cause the kinetics to be slow [18]. The diffusion processes of $\mathrm{H}^{+}$may take place over a much longer time because of the binding effect of $\mathrm{H}^{+}$to acidic groups. In addition, 
it may take a long time to form the hydrogen bonding because the conformational change is required, thus the diameter of the gel decreases very slowly after the completion of the exchange of counter ions [21]. These might be the main reasons for the exceptionally slow kinetics.

\subsection{AFM observations of surface microstructures of dehydrated gels}

The microscopic surface structure of the completely dehydrated gels was directly visualized in air at room temperature by the tapping mode of AFM. Figure 3 presents the height images of the gels synthesized with $0.5,1$, and $2 \mathrm{wt} \% \mathrm{Al}(\mathrm{OH})_{3}$. The gels, which were synthesized in the shape of a plate with $1 \mathrm{~mm}$ and $0.08 \mathrm{~mm}$ thickness, were completely dehydrated in air at room temperature. In this experiment, the gel was not immersed into solvents, and the solvent was not exchanged at all after gelation. Microdomains, which were strongly affected not only by the amount of $\mathrm{Al}(\mathrm{OH})_{3}$ but also by the thickness of the gels, were observed. In order to identify the images quantitatively, the autocorrelation function, $g(r)$, was calculated using the height values obtained from the AFM images $[16,17]$. The data points were found to fit well to a Gaussian distribution, which is scaled by

a decay function, $g(r) / R_{\mathrm{rms}}{ }^{2}-g_{0}=\exp \left[-(r / \xi)^{2}\right]$. In this expression, $g_{0}$ is a constant and corresponds to the incoherent background noise, $R_{\mathrm{rms}}\left(=g(0)^{1 / 2}\right)$ is the root-mean-square roughness, and $\xi$ is the correlation length, which is proportional to the dominated domain size in the AFM image. As is shown in Fig. 4, the domain size decreased and the surface became smooth as the amount of $\mathrm{Al}(\mathrm{OH})_{3}$ was increased. This observation could be attributed to the difference in the degree of the cross-linking density, which should increase with increasing the nominal amount of $\mathrm{Al}(\mathrm{OH})_{3}[18]$. According to the literature [13], the domain structure would be reduced when the cross-linking density is increased, which is consistent with the present observation. These phenomena might constitute an intrinsic property of this system.

On the other hand, the effect of the thickness on the surface microstructure seemed to be extrinsic, since the microscopic structure should be the same in spite of the macroscopic gel size. 
Although the effect was not clearly observed in the roughness analysis (Fig. 4), the surface domain size clearly seemed to depend on the thickness, as shown in Fig. 3. In order to exclude any sample dependence, additional experiments were conducted and are reported in the next section.

\subsection{Effects of the sample thickness on the surface microstructure of dehydrated gels}

The gel was synthesized with $1 \mathrm{wt} \% \mathrm{Al}(\mathrm{OH})_{3}$ and prepared in slabs (their thickness was tapered from 0 to a few millimeters) by two methods. One was the synthesis between two slides in which a thin spacer (thickness is $2 \mathrm{~mm}$ ) was inserted on one edge, and the other was the synthesis in a tilted Petri dish. Therefore, there was a critical difference between the gels in that gelation progressed as it touched the glass or as it was exposed to air. The height images of the completely dehydrated gels measured along with the thickness slopes are shown in Fig. 5. The amplitude as well as the width of the domains in the former was much larger than those of the latter, and the total number of domains is much smaller. Nevertheless, the overall conformations were appreciably same between the gels prepared by two methods; the surfaces of both gels became rough, and the domain became small when the thickness decreased. In order to make clear the formation of the domains in the bulk polymer network, the fractured surfaces of the gels were observed. In this measurement, a thin plate gel, which was prepared between two slides with a spacer of $1 \mathrm{~mm}$ thickness, was fractured along the surface. The fracture started at a notch (a few millimeters deep), which was made on a side surface by a razor blade to localize the stress. Then, one slide was mechanically torn from the other where the notch had propagated in the direction parallel to the top and bottom surfaces, thus forming thin plate gels clamped on slides. The gels were subsequently dehydrated in air at room temperature. Figure 6 shows an example of the height images of the completely dehydrated gels synthesized with $1 \mathrm{wt} \% \mathrm{Al}(\mathrm{OH})_{3}$. One can see that there was no critical difference between the original surface (the gel touched the slide while the gelation progressed) and the fractured surface. These results suggest that the domains might be enhanced during dehydration 
not only in the original surface but also in the bulk network.

The results of roughness analysis calculated from Fig. 5(b) are shown in Fig. 7. As is shown in Fig. 7(a), $R_{\mathrm{rms}}\left(=g(0)^{1 / 2}\right)$ as well as $\xi$ (the inverse of the slope) increased with increasing the thickness of the gel. In Fig. 7(b), $P_{\mathrm{PSD}}(q)$ is plotted vs. $q$ in the log-log scale. $P_{\mathrm{PSD}}(q)$ should be independent of the spatial frequency, $q$, for the lower $q$ range, while it should decrease and tend toward zero when $q$ exceeds the inverse of the characteristic length of the surface. It was clearly observed in this figure that there were two linear relationships with a transition at a characteristic frequency (around 100-200nm). The crossover frequency decreased (the characteristic size increases) with increasing the thickness, which corresponded to the increment of $\xi$. The slope in the respective $q$ range of the larger and smaller of the characteristic frequency did not depend on the thickness. The observed difference in the linearity might be related to the change in the surface dimensionality in the scale range above $200 \mathrm{~nm}\left(q\right.$ range below $\left.5 \times 10^{-3} \mathrm{~nm}^{-1}\right)$.

The present results suggest that the surface roughness grew with time during dehydration both horizontally and vertically, which resulted from the diffusion of aluminum ions. The schematic representation of the network structure is shown in Fig. 8. The inhomogeneous network in assynthesized gels consists of dilute or dense area of polymer density, which might be enhanced during the dehydration. It can be supposed that the domains with densely connected structure can be formed and they can grow as a result of the diffusion of the aluminum ions; the smallest unit of the domains can form a larger structure with time. When the gel is thin, the dehydration is completed before the aluminum ions have completely diffused to form the equilibrium distribution. On the other hand, the diffusion is completed for the thick gels. Therefore, the present observation indicates that there are two mechanisms for the development of the domains, which are not affected by the thickness (introduced intrinsically at gelation) or the dehydration condition (introduced extrinsically during dehydration). In order to interpret the effects of the thickness on the conformation of the characteristic domain, it is necessary to observe the surface structure at higher 
and lower magnifications, where the domain size is uniform for the gels. Furthermore, it is useful to use the phase imaging technique that provides nanometer-scale information about the surface structure often not revealed by the height images, such as variations in composition, adhesion, friction, viscoelasticity, etc. We believe that this is a very important tool for future investigations.

\subsection{Effects of the repeated water exchange on AFM images}

There are two noteworthy properties in the swelling behavior and the surface structure of the dehydrated poly(sodium acrylate) gel. One is that the swelling ratio rapidly increased by the repeated water exchange. This can be attributed to the fact that the excess aluminum ions diffused into the solvent [18], and the counter ions were replaced by protons [21]. The other is that the domain structure depended on the nominal amount of $\mathrm{Al}(\mathrm{OH})_{3}$. On a dehydration process, the aluminum ions have important roles in determining the network structure.

In order to investigate the effects of the water exchange on the surface structure, the solvent was repeatedly exchanged for gels with 4 and $8 \mathrm{wt} \%$, and the surface structure was observed just before the solvent was exchanged. As a result, the macroscopic structure $\left(50 \times 50 \mu \mathrm{m}^{2}\right.$ scale) evidently changed, and the surface became rough. An example is shown in the upper images of Fig. 9 for the gel with $4 \mathrm{wt} \%$. On the other hand, the microdomain can be clearly observed on the microscopic surface $\left(1 \mathrm{X} 1 \mu \mathrm{m}^{2}\right.$ scale), which could not be observed without a water exchange (as is shown in the lower images for the gel with 4 and $8 \mathrm{wt} \%$ ). It is reasonable to assume that the microdomain (around 10-30nm size) was dominant in this system in spite of different nominal amounts of $\mathrm{Al}(\mathrm{OH})_{3}$, which aggregated to form a larger structure (micrometer size) on the dehydrated surface. This assumption is also related with the fact that the macroscopic rough structure was enhanced by the thickness (dehydration condition).

It can be expected that, in the case of the present kind of strongly charged gels, the crosslinking process at gelation itself may produce inhomogeneity due to the repulsive force between 
monomers and the thermal fluctuation of the pre-gel solution. The network structure is not homogeneous by nature but, rather, consists of microdomains [16,22], resulting in the inhomogeneous strain distribution at the microscopic level. It can also be expected in the present system that the domain had a higher density of aluminum ions, which resulted in the existence of a domain structure in the dehydrated gel. The aluminum ions might not be homogeneously incorporated in the main constituent, but they should form many clusters where the density of SA was relatively large. In the presence of excess aluminum ions, the present observation indicates that the macroscopic surface structure evidently changed to a rough surface similar to that of gels with smaller amounts of $\mathrm{Al}(\mathrm{OH})_{3}$, while the microdomains (around 10-30nm) did not change at all. The rough surface might result from the aggregation of the microdomains (extrinsic property), which would be formed at gelation. Although we do not have a quantitative model to explain this picture, the inhomogeneous network structure as well as the aluminum ions should affect the dehydrated surface structure on the microscopic level.

Recently, the appearance of a microphase separation in the dehydrated $\operatorname{poly}(N$ isopropylacrylamide-co-sodium acrylate) (NIPA/SA) gel was found by the SAXS [23-25], and the mechanism is attributed to the inhomogeneous evaporation from the copolymerized network with hydrophobic (NIPA) and hydrophilic (SA) characters having different degrees of interaction with water. It was also suggested that the hydrophilic microdomains could capture the $\mathrm{Na}^{+}$ions and residual water molecules. These observations indicate that the microphase separation may be a universal phenomenon for heteropolymers. On the other hand, the present results indicate that a homopolymer can exhibit a microphase separation during the dehydration process. The condition for the appearance of the domain structure may be the coexistence of at least two intermolecular forces (in the present case, an electrostatic repulsive force and hydrogen bonding) with excess cations. In order to verify these considerations, it is important to make clear the size and existence of the domain structure in the bulk network. Although the surface structure should be reflected by 
the bulk structure, it is difficult to deduce the information on the microstructure of the bulk network from AFM measurements. The experimental observations to give direct information for the bulk network are highly desired on the present samples with different degrees of dehydration. We believe that those results will help to understand quantitatively the swelling behavior observed here.

\section{Conclusion}

We have successfully synthesized the poly(sodium acrylate) gels cross-linked by aluminum ions and investigated some fundamental properties on the swollen states and on the dehydrated states. The swelling ratio of the swollen gels was measured by changing the solvent $\mathrm{pH}$ and adding $\mathrm{NaCl}$ with different concentrations. It was found that each gel swelled at the first stage by repeated solvent exchange, hereafter shrunk to approach the diameter at gelation. These features could be explained using a qualitative model based on the ion exchange and the creation of hydrogen bonding.

The surface microstructure of the dehydrated gels observed by an AFM was characterized by microdomains. The conformations formed by the microdomains strongly depended on the amount of aluminum ions and the gel thickness at gelation. These differences were analyzed in terms of the autocorrelation function and the root-mean-square roughness, which were calculated from the AFM topographies. The surface microstructure of the present system could be determined not only by the concentration of the cross-linker but also by the dehydration conditions. The surface structure of gels with excess aluminum ions was found to be strongly affected by the water exchange.

The present observations, we believe, are of crucial importance not only for understanding physically cross-linked polyelectrolyte gels but also for developing the foundation for future advanced practical applications of gels. 


\section{References}

[1] Tanaka T. Phys Rev Lett 1978;40;820.

[2] Tanaka T, Fillmore DJ, Sun S-T, Nishio I, Swislow G, Shah A. Phys Rev Lett 1980;45; 1636.

[3] Ilavsky M, Plestil J, Dusek K. Eur Polym J 1980;16; 901.

[4] Ricka J, Tanaka T. Macromolecules 1984;18; 83.

[5] Hirokawa Y, Tanaka T. J Chem Phys 1984;81;6379.

[6] Hirotsu S, Hirokawa Y, Tanaka T. J Chem Phys 1987;87; 1392.

[7] Suzuki A, Suzuki H. J Chem Phys 1995;103; 4706.

[8] Mafé S, Manzanares JA, English AE, Tanaka T. Phys Rev Lett 1997;79; 3086.

[9] Liu Y, Velada JL, Huglin MB. Polymer 1999;40; 4299.

[10] Okay O, Sariisik SB. Eur Polym J 2000;36; 393.

[11] Horkay F, Tasaki I, Basser PJ. Biomacromolecules, 2000;1; 84.

[12] Tasaki I. Jpn J Physiol 1999;49; 125.

[13] Abe M, Murase N, Suzuki, T, Eds. Geltechnology, Science Forum Inc., Tokyo, 1997.

[14] Binning G, Quate CF, Gerber C. Phys Rev Lett 1986;56; 930.

[15] Suzuki A, Yamazaki M, Kobiki Y. J Chem Phys 1996;104; 1751.

[16] Suzuki A, Yamazaki M, Kobiki Y, Suzuki H. Macromolecules 1997;30; 2350.

[17] Suzuki A, Kobiki Y, Yamazaki M. Jpn J Appl Phys, Part 1 2003;42; 2810.

[18] According to our quantitative measurement of the diffused amounts of Al ions using an ICPS (Inductively Coupled Plasma Spectrometry), the residual ratio of Al ions in each gel after the repeated water exchanges is in between 80 and $90 \%$ of the nominal amount of $\mathrm{Al}$ ions, and the diffusion of $\mathrm{Al}$ ions into the solvent was completed when the swelling ratio exhibits the maximum. On the other hand, our quantitative measurement of the counter ions using an atomic absorption spectrometry indicates that only $50 \%$ of the original $\mathrm{Na}^{+}$ions diffused into the solvent at the peak of 
the swelling ratio, and the residual $\mathrm{Na}^{+}$ions in the gel continue to diffuse until the diameter becomes almost $d / d_{0}=1$. In addition, the ATR FT-IR spectra of the gels with different degrees of water exchange were obtained, which depended clearly on the degree of the water exchange and confirmed the formation of hydrogen bonding. These results will appear in J. Polym. Sci.B (2005).

[19] Flory PJ, Principles of Polymer Chemistry. Cornell University Press: New York; 1953.

[20] Kawasaki H, Sasaki S, Maeda H. J Phys Chem B 1997;101;5089.

[21] Hirashima Y, Tamanishi H, Sato H, Saito K, Naito A, Suzuki A. J Polym Sci, Part B: Polym Phys 2004;42;1090.

[22] Tanaka T, Sato E, Hirokawa Y, Hirotsu S, Peetermans J. Phys Rev Lett 1985;55;2455.

Phase image

[23] Hirokawa Y, Jinnai H, Nishikawa Y, Okamoto T, Hashimoto T. Macromolecules $1999 ; 32 ; 7093$.

[24] Sugiyama M, Kuwajima S, Soejima Y, Nakamura A, Hiramatsu N, Kikukawa T, Suzuki A, Hara K. Jpn J Appl Phys (Express letter) 1999;38;L1360.

[25] Kuwajima S, Soejima Y, Sugiyama M, Hara K, Nakamura A, Hiramatsu N, Annaka M, Kikukawa T, Suzuki A. Trans Mat Res Soc Jpn 2000;25;747. 


\section{Figure Captions}

Fig. 1.

Change in the swelling ratio, $d / d_{0}$, of poly(sodium acrylate) gels crosslinked by aluminum ions $(4 \mathrm{wt} \%)$ at room temperature by the repeated exchanges of solvent (pure water) at a constant interval (24hours). Effects of $\mathrm{pH}(\mathrm{a})$, and $\mathrm{NaCl}$ concentration (b).

\section{Fig. 2.}

Time evolution of the swelling ratio, $d / d_{0}$, of poly(sodium acrylate) gels crosslinked by aluminum ions $\left(\mathrm{Al}(\mathrm{OH})_{3} ; 2,4\right.$ and $\left.8 \mathrm{wt} \%\right)$ at room temperature. The diameter of the gel was measured when the respective gel takes the equilibrium diameter after the solvent was exchanged (not at a constant interval).

\section{Fig. 3.}

AFM images of the plate gels synthesized with different amounts of $\mathrm{Al}(\mathrm{OH})_{3}(0.5,1$ and $2 \mathrm{wt} \%)$. The thickness at gelation of the gel is $1 \mathrm{~mm}$ (a) and $0.08 \mathrm{~mm}(\mathrm{~b})$, which was completely dehydrated in air at room temperature. The vertical scales have been 2 times exaggerated to display clearly the spatial amplitude in the $z$-direction.

\section{Fig. 4.}

Dependence of $R_{\text {rms }}$ (circles) and $\xi$ (triangles) on the amount of $\mathrm{Al}(\mathrm{OH})_{3}$, calculated by the AFM images in Fig. 3. The open and closed symbols denote the data from the gels with 1 and $0.08 \mathrm{~mm}$, respectively. The solid lines are to guide the eye. 


\section{Fig. 5.}

AFM images of the completely dehydrated gels (crosslinked by aluminum ions; $1 \mathrm{wt} \% \mathrm{Al}(\mathrm{OH})_{3}$ ) synthesized by two different methods (schematically illustrated); the gelation progresses while touching the glass (a) or exposing to the air (b). The images were measured along with the thickness slopes .

\section{Fig. 6.}

An example of the AFM images of a completely dehydrated gel (crosslinked by aluminum ions; $\left.1 \mathrm{wt} \% \mathrm{Al}(\mathrm{OH})_{3}\right)$; the original surface (the gel touched the glass while the gelation progressed) and the fractured surface.

\section{Fig. 7.}

Roughness analysis of the AFM images in Fig. 5(b); (a) the autocorrelation functions, and (b) surface power spectral density as a function of spatial frequency calculated from the autocorrelation functions.

\section{Fig. 8.}

Schematic representation of the network structure of the poly(sodium acrylate) gels cross-linked by aluminum ions; an as-synthesized gel (inhomogeneous) (a), and a dehydrated thin gel (b), and a thick gel (c).

\section{Fig. 9.}

Effects of water exchange on the surface structure where the solvent was repeatedly exchanged for the gels crosslinked by aluminum ions; 4 and $8 \mathrm{wt} \% \mathrm{Al}(\mathrm{OH})_{3}$. 

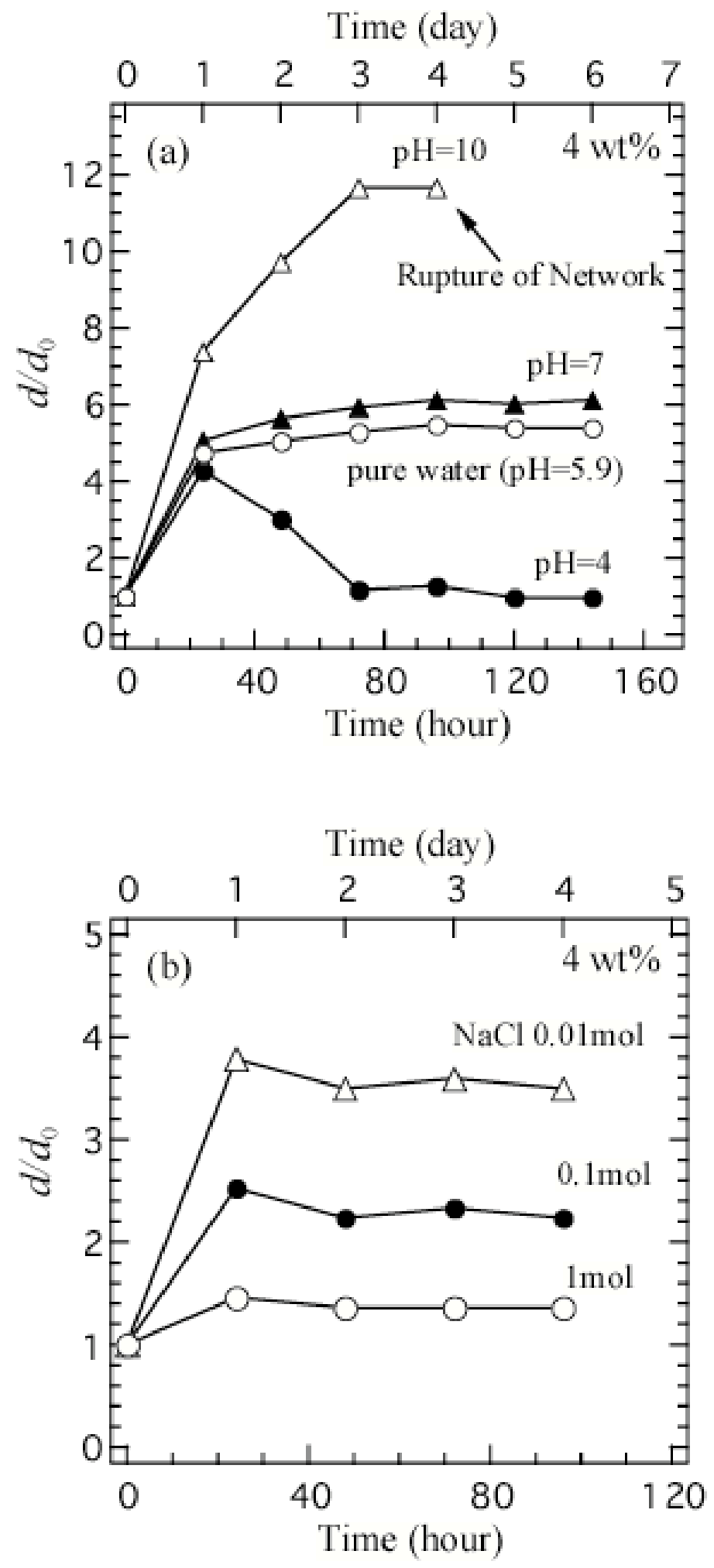

Fig.1 Harada et al. 


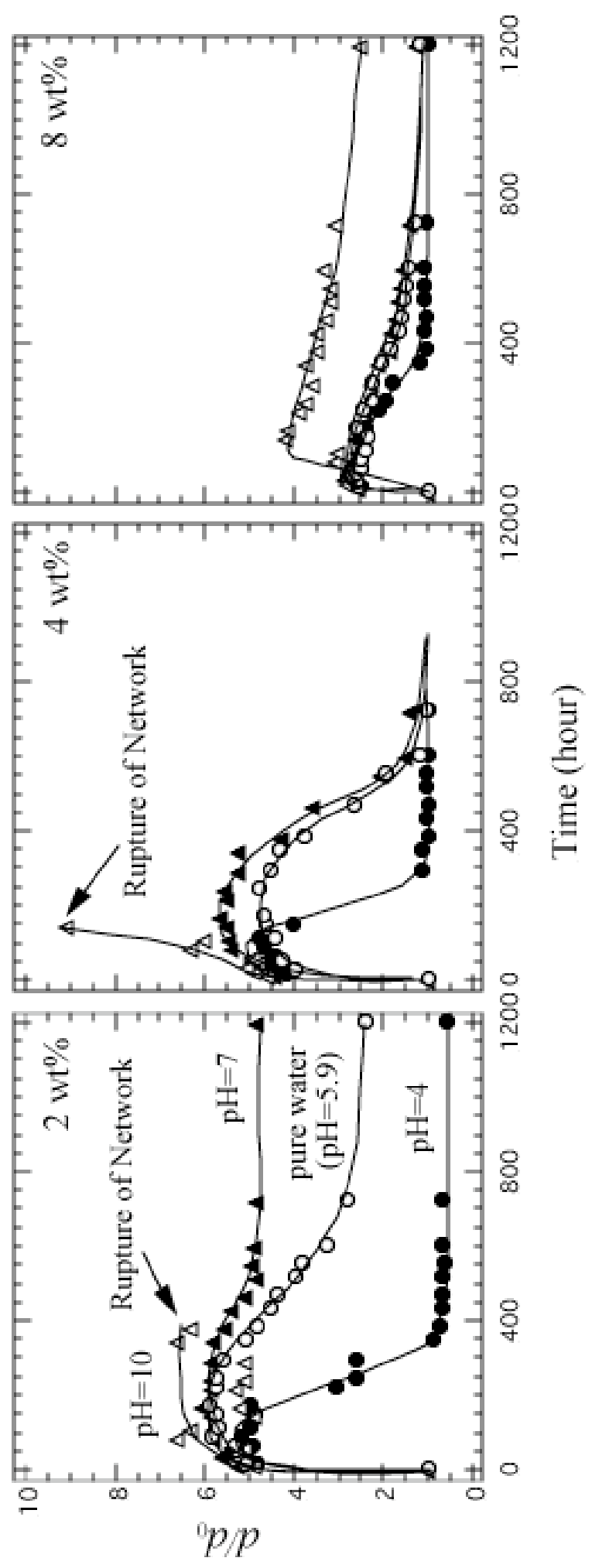

Fig.2 Harada et al. 

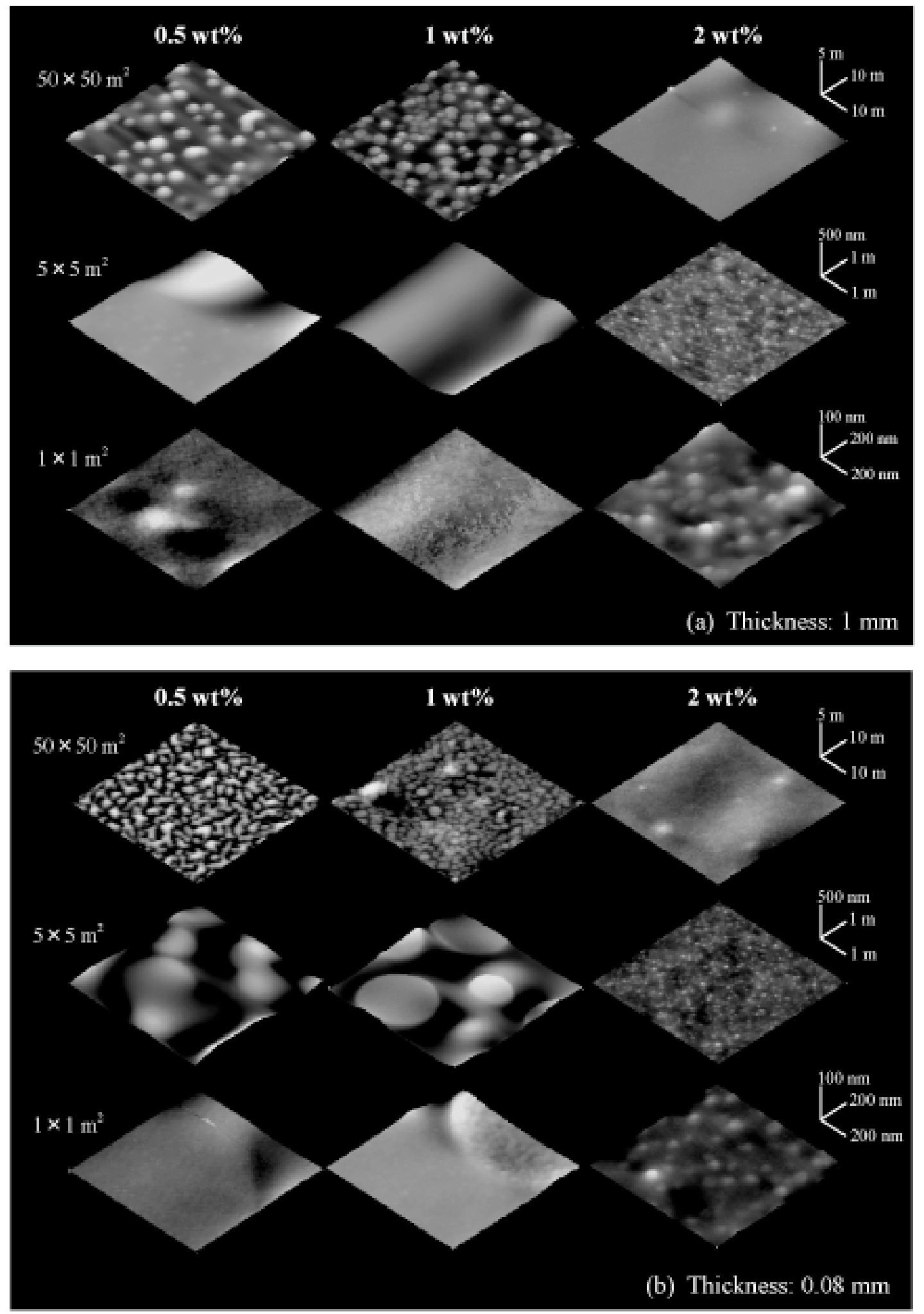

Fiq. 3 Harada, 


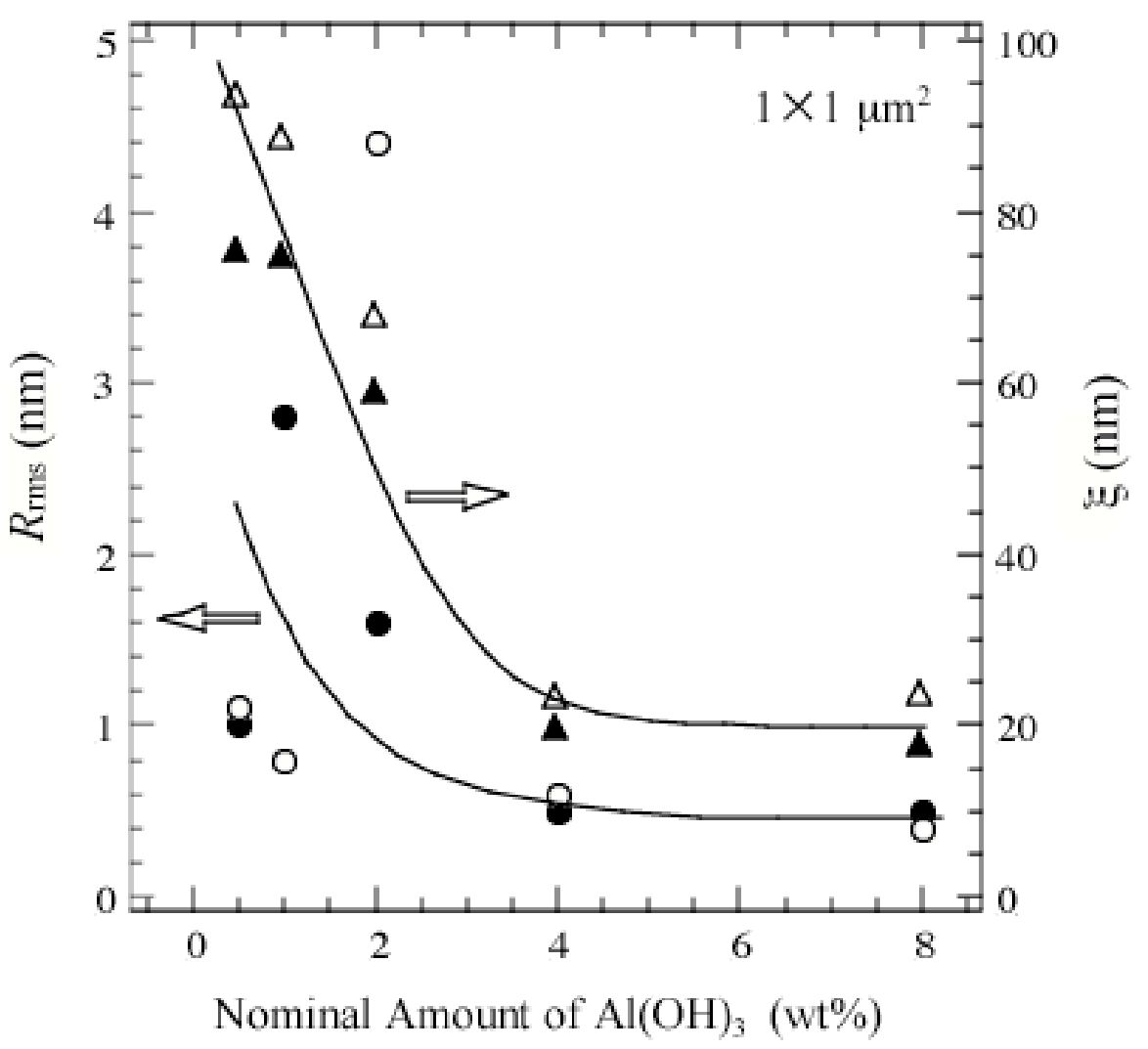

Fig.4 Harada et al. 


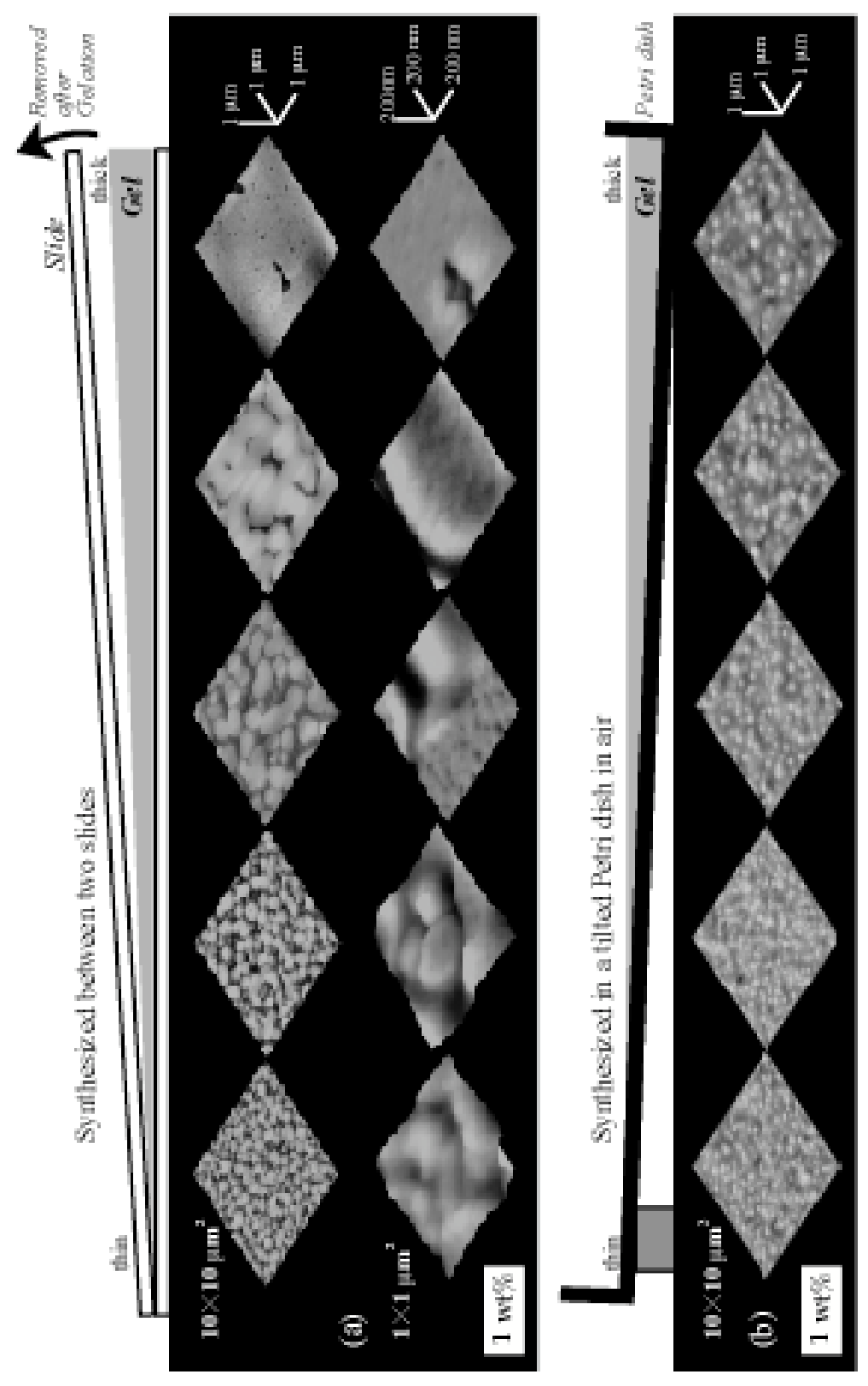

Fig. 5 Harada, et al. 


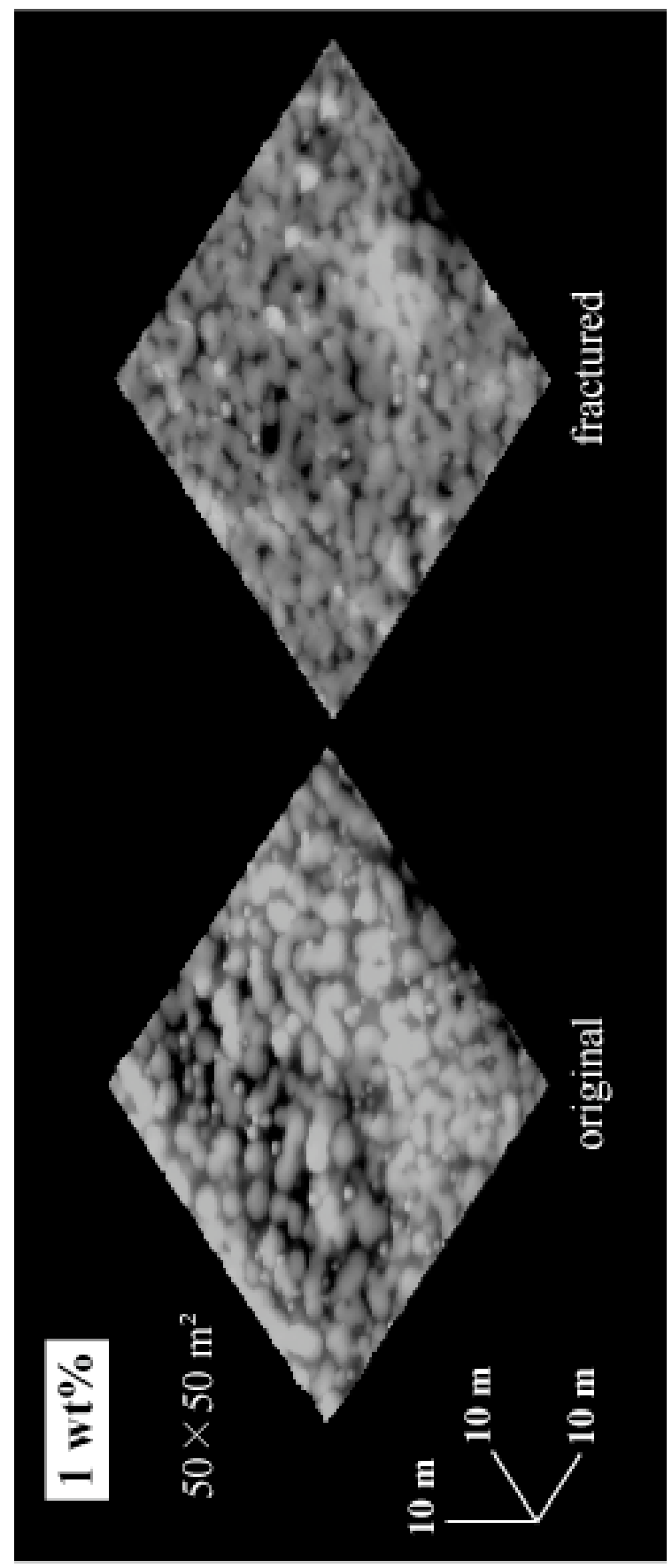

Fig. 6 Harada, et al. 

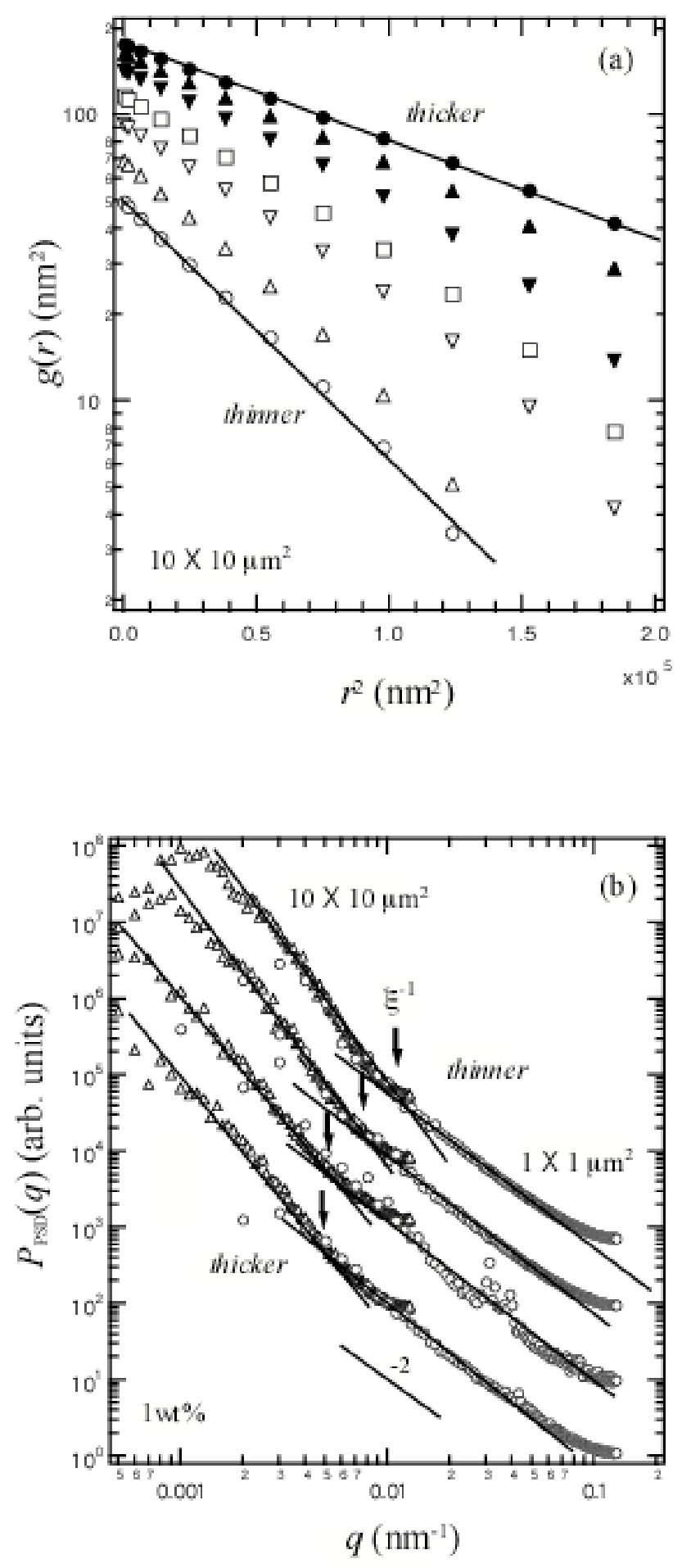

Fig. 7 Harada et al. 


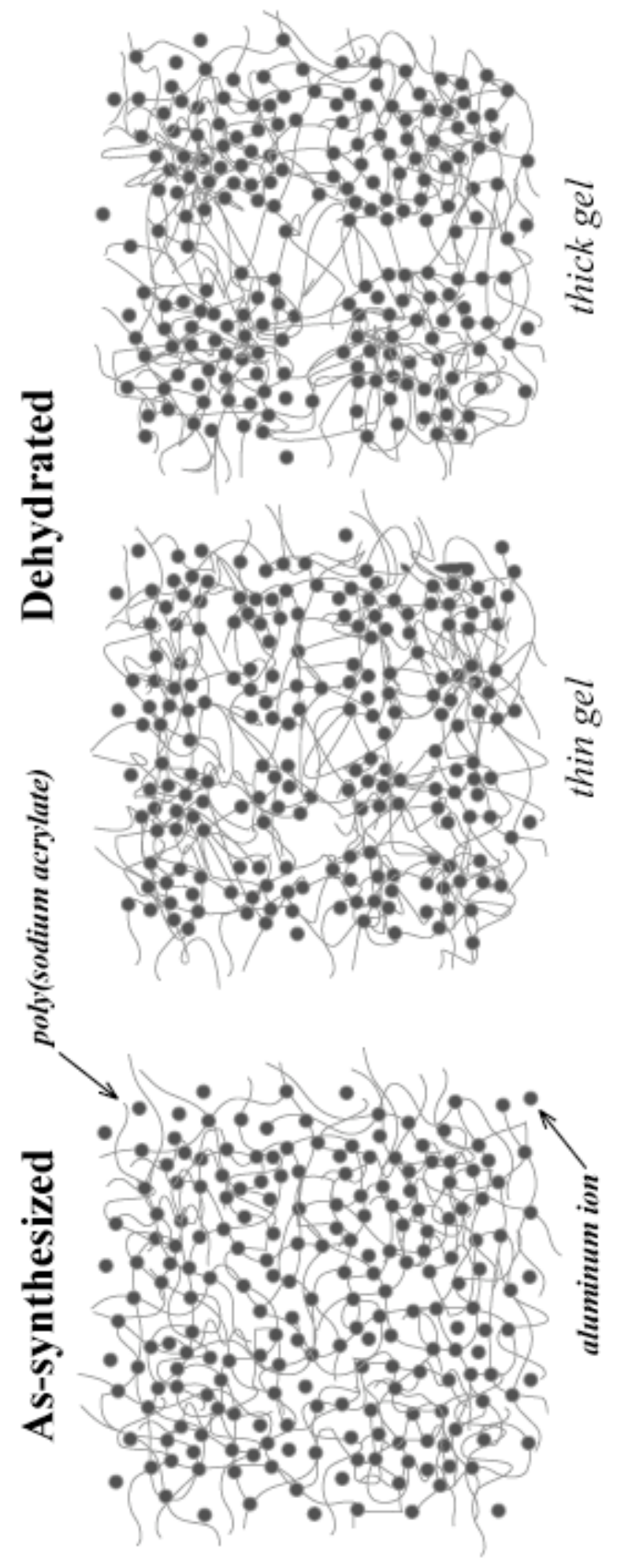

Fig. 8 Harada, et al. 


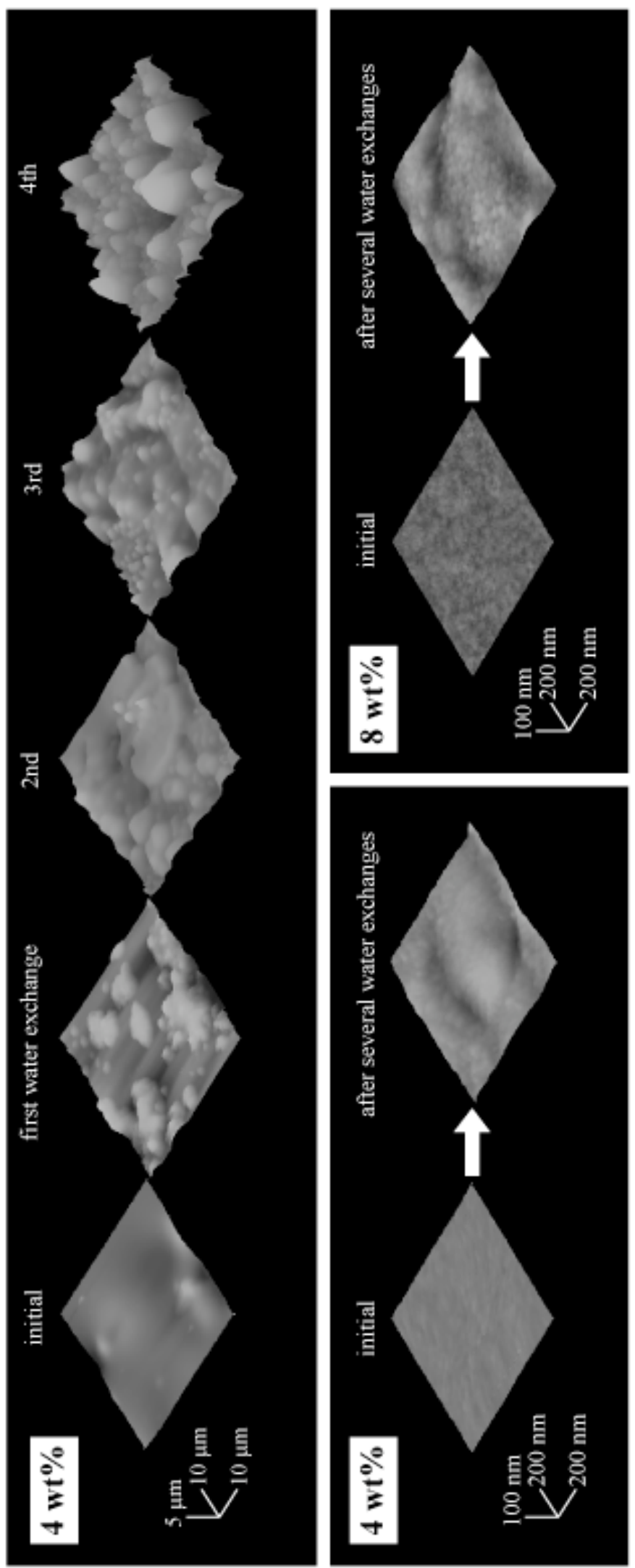

Fig.9 Harada, et al. 